\title{
Growth and Characterization of Pure and L-Phenylalanine doped Potassium Hydrogen Phthalate Crystals
}

\author{
T. Karpagam ${ }^{1}$, K. Balasubramanian ${ }^{2}$ \\ ${ }^{1}$ Research Scholar, $P G \&$ Research Department of Physics, The M.D.T. Hindu College, India \\ ${ }^{2} P G \&$ Research Department of Physics, The M.D.T. Hindu College, India
}

\begin{abstract}
Semi Organic Potassium Hydrogen Phthalate single crystal with amino acid L-Phenylalanine as a dopant was grown by slow evaporation technique. The grown crystals were subjected to powder XRD analysis, and the result confirms that the crystals were belongs to orthorhombic structure. The Fourier Transform Infrared Spectroscopy study confirms the incorporation of L-Phenylalanine in to Potassium Hydrogen Phthalate crystal. The optical transparency of the grown crystal was studied by UV-Visible spectrophotometer. Mechanical strength of the grown crystal was estimated by Vickers hardness Test. The dielectric constant and dielectric loss has been studied as a function of frequency for the doped crystals.
\end{abstract}

Keywords: Dielectric studies, Infrared spectroscopy, Micro hardness; Optical transmittance studies, X-ray diffraction

\section{Introduction}

In recent years, the search of NLO materials with good transparency has lead to the development of photonic and optoelectronic technologies [1]. Potassium Hydrogen Phthalate, often called simply KHP is an interesting analyzer material in X-Ray spectroscopy $[2,3]$. KHP possesses piezoelectric, pyroelectric, elastic and non linear optical properties. KHP crystallizes into orthorhombic system with the non-centrosymmetric space group $p_{\text {ca21 }}$ and the lattice parameters are found to be $a=9.605 \mathrm{~A}^{0}, b=13.331 \mathrm{~A}^{0}, c=6.473 \mathrm{~A}^{0}$. The perfect cleavages (010) plane of KHP is more suitable for surface morphological studies like etching and SEM. Recently KHP crystals have been used as substrate materials for the growth of a highly oriented film of conjugated polymers with high non linear susceptibility [4]. Now amino acid family crystals are also playing an important role in the field of non-linear optics. In the present work, the structural, optical and thermal properties of amino acid L-Phenylalanine doped KHP crystal, which was grown by slow evaporation technique at room temperature.

\section{Experimental Method}

The amino acid L-Phenylalanine doped KHP crystals were grown by the slow evaporation solution growth method. The KHP salt was dissolved in de ionized water. The solution was stirred well for an hour constantly using magnetic stirrer. With this solution, 0.05 and $0.1 \mathrm{~mol} \%$ of L-Phenylalanine was added as a dopant. After homogeneous mixing solutions, it was kept in dust free area for slow evaporation. After a period of time a good quality transparent single crystals of undoped and L-Phenylalanine doped KHP crystals were grown. Photographs of grown doped and undoped crystal are shown in Fig 1.

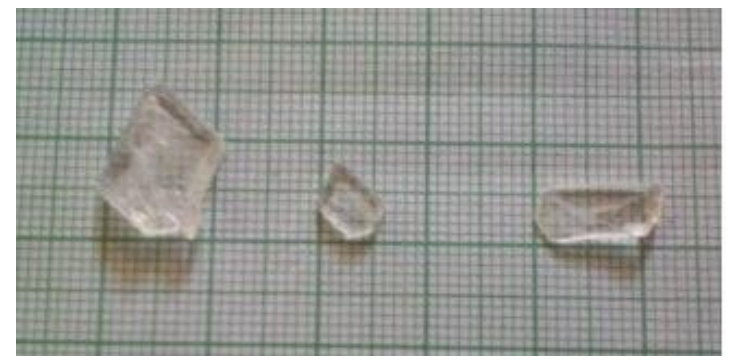

Fig.1. Grown crystals of undoped and $(0.05 \& 0.1 \mathrm{~mol} \%)$ L-Phenylalanine doped KHP crystals 


\subsection{Powder XRD Analysis}

\section{Results And Discussion}

The grown crystals were subjected to powder XRD analysis using X'pert pro with cu K $\alpha 1$ radiation $\left(\lambda=1.54060 \mathrm{~A}^{0}\right)$ for the phase analysis. Powder XRD patterns of the grown crystals shown in Fig. 2 . The results confirmed that all the crystals formed in orthorhombic structure with space group Pca $_{21}$ according to JCPDS data (31-1855). The XRD pattern of L-Phenylalanine doped KHP shows slight changes in peak intensities and peak positions, when compared to the undoped KHP [5]. The cell parameters of undoped and doped KHP crystals were calculated and the data are given in table 1.

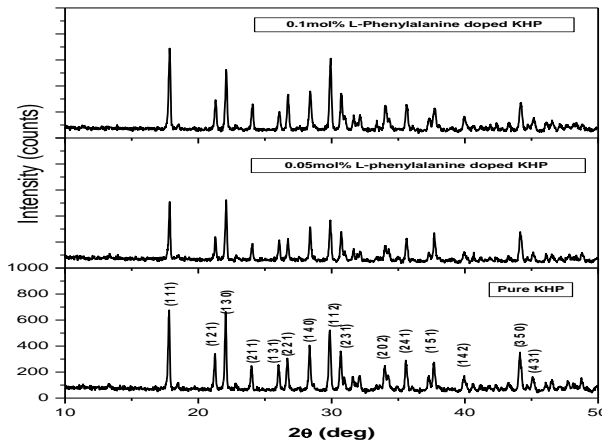

Fig.2. XRD pattern for undoped and $(0.05 \& 0.1 \mathrm{~mol} \%)$ L-Phenylalanine doped KHP crystals

Table 1: Lattice parameters of undoped and doped KHP crystals

\begin{tabular}{|l|l|c|c|c|}
\hline & KHP & KHP & $\begin{array}{c}0.05 \mathrm{M} \% \\
\text { L-Phenylalanine } \\
\text { doped KHP }\end{array}$ & $\begin{array}{c}0.1 \mathrm{M} \% \\
\text { L-Phenylalanine } \\
\text { doped KHP }\end{array}$ \\
\hline & 9.605 & 9.625 & 9.6225 & 9.6025 \\
\hline & 13.331 & 13.3195 & 13.3037 & 13.2984 \\
\hline & 6.472 & 6.4603 & 6.4413 & 6.4378 \\
\hline & 828.8305 & 828.2139 & 824.8381 & 822.6946 \\
\hline
\end{tabular}

\subsection{FTIR Analysis}

FTIR spectrum of undoped and L-Phenylalanine doped KHP crystals were recorded using Perkin Elmer spectrum in the range $400-4000 \mathrm{~cm}^{-1}$ by $\mathrm{KBr}$ pellet technique. The FTIR spectra of the grown crystals are given in Fig.3. The FTIR spectra of 0.05 and $0.1 \mathrm{~mol} \%$ doped crystals show strong NH symmetric stretching at $2400-2650 \mathrm{~cm}^{-1}$. More NH stretching vibrations are introduced due to doping of L-Phenylalanine and the $\mathrm{NH}$ absorption peak become stronger. In the FTIR spectrum, $\mathrm{OH}^{-}$stretching hydrogen bond appears at $2784 \mathrm{~cm}^{-1}$ for the undoped and $2794 \mathrm{~cm}^{-1}, 2791 \mathrm{~cm}^{-1}$ for the doped compound. This shift is due to the incorporation of L-Phenylalanine into the KHP material. This shift may also be due to the free stretching of $\mathrm{NH}_{2}$ group present in the dopant. In addition to that, $\mathrm{C}=\mathrm{C}$ ring stretching appears at $1489 \mathrm{~cm}^{-1}$ for undoped and $1483 \mathrm{~cm}^{-1}, 1482 \mathrm{~cm}^{-1}$ for the dopant .

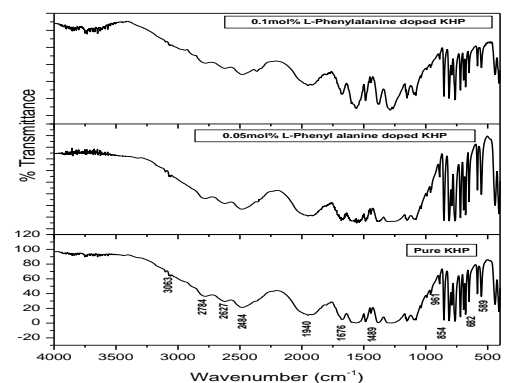

Fig.3. FTIR analysis for undoped and $(0.05 \& 0.1 \mathrm{~mol} \%)$ L-Phenylalanine doped KHP crystals

The UV-visible - NIR spectroscopy was performed on the samples by using UV-700 SHIMADZU spectrophotometer. The recorded transmittance spectra of undoped and doped crystals in the wavelength range 200-1100 $\mathrm{nm}$. Large absorptions are found at around $300 \mathrm{~nm}$ for undoped and doped KHP crystals due to $n-\pi$ transition of the carbonyl group of the carboxyl functions $[6,7]$. There is no absorption observed in the region from 350-1100 nm for the undoped and doped crystals which makes the materials suitable for second harmonic generation. The recorded spectra are shown in Fig.4. 


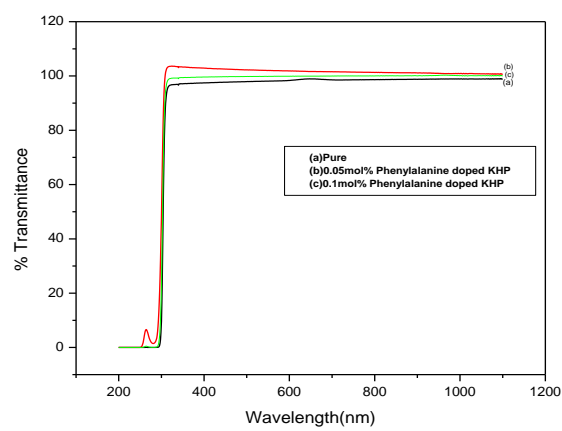

Fig.4. UV Transmittance spectra of undoped and L-Phenylalanine doped KHP crystals

\subsection{Micro hardness Test}

To estimate mechanical hardness, the indentation hardness is measured as the ratio of applied load to the surface area of the indentation. A plot drawn on hardness value and corresponding load is shown in Fig.5. The hardness number was calculated using the relation, $\mathrm{Hv}=1.8544 \mathrm{P} / \mathrm{d}^{2} \mathrm{Kg} / \mathrm{mm}^{2}$. Where, $\mathrm{Hv}$ is the Vickers hardness number, $\mathrm{P}$ is the applied load and $\mathrm{d}$ is the diagonal length of the indentation impression. It is observed that the hardness number increases with increase in load and it reveals that the doped KHP crystal exhibits reverse indentation effect. The plot of $\log (\mathrm{P})$ against $\log (\mathrm{d})$ is almost a straight line as shown in Fig.6. From the slope of this plot the work hardening coefficient is found to be greater than 2 for doped crystals. Onitsch states that the values $1.0<n<1.6$ for hard materials and $n>1.6$ for soft materials [8-10]. The work hardening coefficient of L- Phenylalanine doped KHP values are $n=2.47$ and $n=2.76$. Hence, it is concluded that doped crystals are also soft materials.
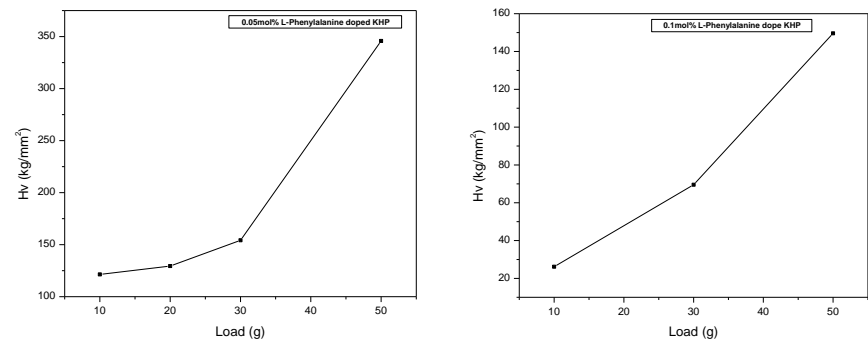

Fig.5. Vickers hardness vs. Load of L-Phenylalanine doped KHP crystals
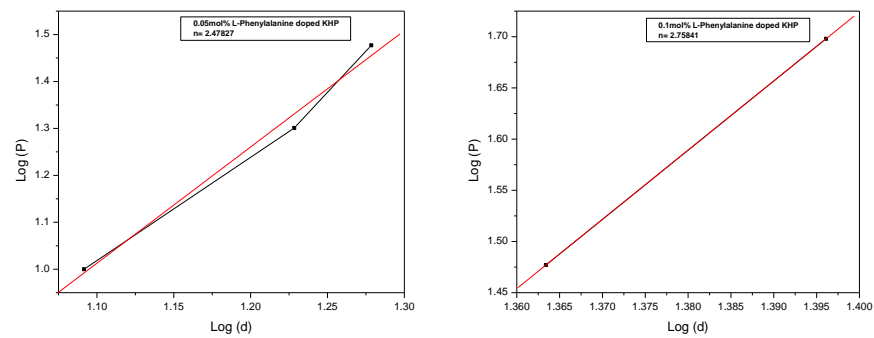

Fig.6. The plot Log (P) against log (d) for L-Phenylalanine doped KHP crystals

Dielectric measurement is one of the useful methods for characterization of electrical response in crystalline material. Fig.7. shows the variation of dielectric constant and dielectric loss of the undoped and doped crystals at different temperatures at constant frequency. The decrease in dielectric constant of the undoped and doped crystal at low frequencies may be attributed to the contribution of electronic, ionic, orientation and space charge polarizations which depend on the frequencies. It is further observed that both dielectric constant and dielectric loss decreases with increase in temperature. 

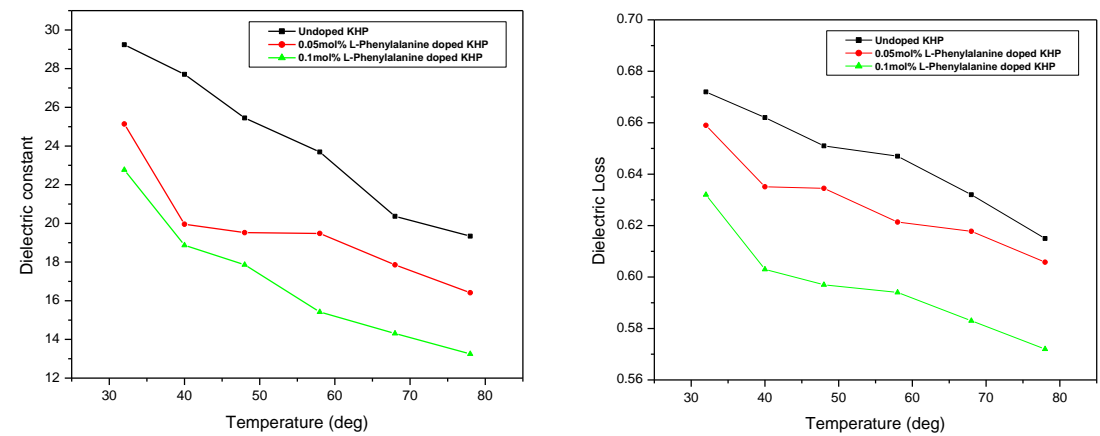

Fig.7. The variation of dielectric constant and dielectric loss at different temperatures for undoped and L-Phenylalanine doped KHP crystals

\section{Conclusion}

The undoped and L-Phenylalanine doped KHP crystals were grown by slow evaporation method. XRD studies for the grown crystals have been carried out and it is found that the structures of the grown crystals are orthorhombic. The unit cell parameters of the grown crystals were obtained from XRD studies. The various functional groups and the modes of vibrations were identified by FTIR spectroscopy. From the UV-Visible spectral analysis of the grown KHP crystals, a strong absorption is observed at $300 \mathrm{~nm}$ for all grown crystals and the energy gap is $6.7 \mathrm{eV}$. Micro hardness measurements imply that the pure and doped crystals come under the soft materials category. From the dielectric study, it is found that both dielectric constant and dielectric loss of the crystal decreases with increase in temperature.

\section{Acknowledgement}

The authors are grateful to the Management of The M.D.T Hindu College, Pettai, Tirunelveli, for providing to access the DST-FIST Sponsored Instrumentation Laboratory and Research facilities of the Department.

\section{References}

[1]. M. Lydia caroline, S. Vasudevan, curr.Appl.Phys.9 (2009), p.1054

[2]. D.R.Kanis, M.A.Ratner, T.S.Marks, chem.Rev.94(1994) 195

[3]. J.George, S.K.Premchandran,J.Phys, D 14 (1981) 1277

[4]. S. Parthiban,S. Murali, G. Mathurammbal, S.P. Meenakshisundaram, S.C. Mojumdar, j.Therm.Anal.Calorim.100(2010) 751-756

[5]. N. Balamurugan, M.Lenin, P.Ramasamy, Mater, Lett,61(2007)1896

[6]. P.Rajesh, P.Ramasamy, Physica B 404(2009) 1611

[7]. K. Sangwal, Additives and crystallization Process, john wiley and sons Ltd, 2007.

[8]. E.M. Onitsch, Mikroskopie 2 (1947) 131-151.

[9]. Kumaresan, P; Moorthy babu, Anbarasan, P.M; optical materials. 2008, 30, 1361-1368

[10]. Elakkina Kumaran, A; Kanchana, P; Sekar, C: Spectrochimica acta part A: Molecular and Biomolecular Spectroscopy. 2012, 91, 370-374. 